\title{
INTEGRABILITY OF CHERN-SIMONS-HIGGS VORTEX EQUATIONS and A REDUCTION OF THE SELF-DUAL YANG-MILLS EQUATIONS TO THREE DIMENSIONS
}

\author{
Jeremy Schiff \\ Physics Department \\ Columbia University \\ New York \\ NY 10027
}

\section{INTRODUCTION}

I would like to make brief presentations on two topics, both of which focus on an issue of 'integrability' in equations of interest in high energy physics. In my first talk, I would like to introduce the Chern-Simons-Higgs vortex equations, which describe classical solutions of a certain $(2+1)$-dimensional field theory. In flat spacetime these equations are non-integrable, but in curved spacetime the ODE describing cylindrically symmetric vortices can, by correct choice of the metric, be made to be a degenerate case of the third Painlevé equation, possessing rational solutions which can be written down. Remarkably, the overall features of the solutions (found numerically) for the flat spacetime case, are very similar to those found in the integrable case, suggesting that maybe the non-integrable case should be looked on as a "perturbation' of the integrable case. My second talk is on the topic of a reduction of the self-dual Yang-Mills equations from four to three dimensions: there has been considerable interest recently in the self-dual Yang-Mills equations as a 'master equation', from which many integrable systems can be obtained by suitable reductions. Here I focus on a method to reduce to three dimensions, but the systems that emerge are really trivial generalizations of two dimensional integrable systems.

For the reader's ease, equations and references in my first talk are referred to by letter $(\mathrm{A}, \mathrm{B}, \mathrm{C}, \ldots)$, and in my second talk by number $(1,2,3, \ldots)$.

\section{INTEGRABILITY OF CHERN-SIMONS-HIGGS VORTEX EQUATIONS}

It has been known for some years that in $(2+1)$ dimensions there exists an interesting alternative to standard electromagnetism $[\mathrm{A}]$. In $(d+1)$ dimensions, standard electromagnetism is defined by the action

$$
S=\int d^{d+1} x \sqrt{g}\left(-\frac{1}{4} F_{\mu \nu} F^{\mu \nu}\right)
$$

Here we understand that the fundamental quantity is the potential $A_{\mu}(x), \mu=$ $0,1,2, \ldots, d$, and $F_{\mu \nu}=\partial_{\mu} A_{\nu}-\partial_{\nu} A_{\mu}$. In writing this we have allowed for the possibility that we are working on a curved spacetime, with metric tensor $g_{\mu \nu}$ (for flat spacetime $\left.g_{\mu \nu}=\operatorname{diag}(1,-1,-1, \ldots,-1)\right) ; F^{\mu \nu}$ is defined by $F^{\mu \nu}=g^{\mu \mu^{\prime}} g^{\nu \nu^{\prime}} F_{\mu^{\prime} \nu^{\prime}}$ where $g^{\mu \nu}$ is the inverse of the metric tensor (i.e. $g^{\mu \nu} g_{\nu \rho}=\delta_{\rho}^{\mu}$ ), and $g=\operatorname{det}\left(g_{\mu \nu}\right)$. If $d=2$ we can 
consider an alternative action

$$
S=\int d^{3} x\left(-\frac{1}{4} \alpha \sqrt{g} F_{\mu \nu} F^{\mu \nu}+\frac{1}{4} \kappa \epsilon^{\mu \nu \rho} F_{\mu \nu} A_{\rho}\right)
$$

where $\alpha, \kappa$ are constants. The extra term is known as the Chern-Simons term, and the theory based on this action (with non-vanishing $\alpha, \kappa$ ) is called 'topologically massive electromagnetism'; the photon in this theory has mass, due to the Chern-Simons term, which is independent of the metric tensor $g_{\mu \nu}$, and therefore topological.

If in (B) we set $\alpha=0$ we obtain 'pure Chern-Simons electromagnetism'. The equations of motion (i.e. the variational equations $\delta S=0$ ) in this theory are $F_{\mu \nu}=0$, and from these it follows that, at least within a topologically trivial region of spacetime, there is no classical dynamics. Somewhat surprisingly though, if one couples pure Chern-Simons electromagnetism to a certain type of other field then the electromagnetic field does become dynamical [B]. Specifically, let $\phi(x)$ be a complex scalar field and consider the action

$$
S=\int d^{3} x\left(\frac{1}{4} \kappa \epsilon^{\mu \nu \rho} F_{\mu \nu} A_{\rho}+\sqrt{g} D_{\mu} \phi D^{\bar{\mu}} \phi-\sqrt{g} V\left(|\phi|^{2}\right)\right)
$$

where $D_{\mu} \phi=\left(\partial_{\mu}+i e A_{\mu}\right) \phi$ ( $e$ is the 'coupling constant'), and $V\left(|\phi|^{2}\right)$ is some 'Higgs potential', that is, a function with a minimum at some value of $|\phi|^{2}$ other then 0 . This is the general (abelian) Chern-Simons-Higgs action, and an action of this type is thought to play a role in high- $T_{c}$ superconductivity.

Following [C], we shall consider a particular Chern-Simons-Higgs action, with the potential

$$
V\left(|\phi|^{2}\right)=\frac{e^{4}}{k^{2}}|\phi|^{2}\left(|\phi|^{2}-m\right)^{2}
$$

If further we take the metric to have the form $g_{\mu \nu}=\operatorname{diag}(1,-b,-b)$ where $b$ is some function of $x^{1}, x^{2}$, the space variables, then it is possible to show a remarkable fact: while the equations of motion for the action $(C)$ include a second order PDE (the equation of motion for the scalar field), for the particular choice (D) it is possible to obtain finite energy, stationary (i.e. $x^{0}$ independant) solutions of the equations by solving a set of only first order PDEs. For full details see $[\mathrm{C}],[\mathrm{D}]$; here I wish to focus on integrability properties of the final equations, so I just quote the result. For any stationary solution it is easy to show that

$$
A_{0}=\frac{-\kappa F_{12}}{2 b e^{2}|\phi|^{2}}
$$

The system of equations to be solved for the remaining fields $\phi, A_{i}, i=1,2$, is

$$
\begin{gathered}
\left(D_{1} \pm i D_{2}\right) \phi=0 \\
e F_{12}= \pm \frac{2 e^{4}}{\kappa^{2}} b|\phi|^{2}\left(|\phi|^{2}-m\right)
\end{gathered}
$$

(where it is understood that one has to choose the upper or lower signs consistently in both equations). This system is clearly underdetermined, due to the gauge freedom $A_{i} \rightarrow A_{i}+\partial_{i} \Lambda, \phi \rightarrow e^{-i \Lambda} \phi$. For solutions given by equations (F) the energy is given by

$$
\begin{gathered}
E=\mp m e \Phi \\
\Phi=\int d^{2} x F_{12}
\end{gathered}
$$

Since the energy is positive, from (G1) it follows that the quantity $\Phi$ (the flux) must be negative (positive) for the choice of upper (lower) signs in equations $(\mathrm{F})$; for all 
the solutions we shall look at here $|\phi|^{2}$ is bounded above by $m$, so this is indeed the case, from equation (F2). The question as to whether the first order system (F) yields all finite energy, stationary solutions of the equations of motion (for our choice of potential and the flat metric) is currently open.

Equation (F1) can be solved to give $A_{i}$ in terms of $\phi$. Parametrizing $\phi$ by

$$
\phi=\sqrt{m \chi} e^{-i \omega}
$$

where $\chi, \omega$ are real functions of $x^{1}, x^{2}$ with $\chi$ nonnegative, we obtain

$$
e A_{i}=\mp \frac{1}{2} \epsilon_{i j} \partial_{j} \ln \chi+\partial_{i} \omega
$$

Substituting in (F2) we obtain one equation for $\chi$ (the function $\omega$ in $(\mathrm{H}),(\mathrm{I})$ is just the gauge degree of freedom):

$$
\nabla^{2} \ln \chi=\gamma^{2} b \chi(\chi-1)
$$

where

$$
\gamma=\frac{2 e^{2} m}{\kappa}
$$

We seek solutions to $(J)$ which are 'well-behaved' everywhere, and for which $\chi \rightarrow 0$ or $\chi \rightarrow 1$ at spatial infinity (corresponding to $|\phi|^{2}$ tending to one of the two vacuum values 0 or $m$, necessary for finite energy).

I will return to equation $(\mathrm{J})$ briefly later, but the main point I would like to make can be well-illustrated by restricting to cylindrically symmetric solutions; specifically if $b$ is a function of $r=\sqrt{x_{1}^{2}+x_{2}^{2}}$ alone, then it makes sense to look for solutions of $(\mathrm{J})$ where $\chi$ is a function of $r$ alone. We then obtain the ODE

$$
\frac{1}{r}\left(\frac{r \chi^{\prime}}{\chi}\right)^{\prime}=\gamma^{2} b \chi(\chi-1)
$$

I will present solutions to this for the flat space case $(b=1)$, when the equation is non-Painlevé, and for the case $b \propto 1 / r^{2}$, when the equation is Painlevé. Interestingly, the overall features of the solutions are very similar for both cases, suggesting that maybe they can be regarded as 'perturbations' of each other.

For $b=1$ there are four types of (well-behaved) solutions, found in $[\mathrm{C}]$ by a combination of analytic and numerical techniques (the solutions have not been obtained in closed form).

1. Vacuum solutions, $\chi=0$ and $\chi=1$.

2. 'Vortex solutions', $\chi(0)=0, \chi \rightarrow 1$ as $r \rightarrow \infty$. For any positive integer $n$ there is a solution of this type, with $\chi \sim G_{n}(\gamma r)^{2 n}$ for small $r$, where $G_{n}$ is some fixed number depending on $n$, and with $(1-\chi)$ decaying as a multiple of $K_{0}(\gamma r)$ for large r. These solutions have flux $\Phi=2 \pi n / e$.

3. Solutions with $0<\chi(0)<1$, and $\chi \rightarrow 0$ as $r \rightarrow \infty$. There is a one parameter family of such solutions, and $\chi(0)$ can be taken to be the parameter. For large $r$, the solutions decay as $(\gamma r)^{-2 \alpha}$ for some positive number $\alpha$ determined by $\chi(0)$. The flux is $\Phi=2 \pi \alpha / e$.

4. Solutions with $\chi(0)=0$ and $\chi \rightarrow 0$ as $r \rightarrow \infty$. These solutions are specified by two parameters, a positive integer $n$ and a number $H$ with $0<H<G_{n}$; for small $r, \chi \sim H(\gamma r)^{2 n}$, and for large $r, \chi$ decays as $(\gamma r)^{-2 \alpha}$ where the number $\alpha$ is determined by $n, H$. The flux is $\Phi=2 \pi(n+\alpha) / e$.

In the above list the fact that $n$ is an integer is not required if we just wish to solve equation (L); it arises from the requirement that $A_{i}$ should be single-valued at the origin. So essentially we have 1-parameter families of types 2 and 3 , and 
a two parameter family of type 4 (the different 'types' classify different asymptotic behaviors).

We now look at the case of equation (L) with $b=4 p^{2} / \gamma^{2} r^{2}$ (this case was suggested by Painlevé analysis of (L) as will be explained later). Here $p$ is a positive constant. We obtain the equation

$$
\left(\frac{r \chi^{\prime}}{\chi}\right)^{\prime}=\frac{4 p^{2}}{r} \chi(\chi-1)
$$

which is a special case of the third Painlevé equation, with rational solutions, found in [E]. Again we can write down four types of solution.

1. Vacuum solutions, $\chi=0, \chi=1$.

2. Vortex solutions. For any $\alpha>0$ there is a solution, with $\chi(0)=0, \chi(\infty)=1$ given by

$$
\chi=\frac{1}{1+\alpha r^{-2 p}}
$$

with $\Phi=2 \pi p / e$.

3. Solutions with $\chi(1)=1, \chi(\infty)=0$. For any $\beta>0$ there is such a solution, given by

$$
\chi=\frac{1}{1+\beta r^{2 p}}
$$

with $\Phi=2 \pi p / e$

4. Solutions with $\chi(0)=\chi(\infty)=0$. For any $\alpha, \beta>0$ we have a solution

$$
\chi=\frac{\mu}{\left(\alpha+r^{2 \lambda}\right)\left(\beta+r^{-2 \lambda}\right)}
$$

Here $\mu, \lambda$ are defined in terms of $p, \alpha, \beta$ by

$$
\begin{gathered}
\alpha \beta=\frac{p+\lambda}{p-\lambda} \\
\mu=\frac{2 \lambda^{2}}{p(p+\lambda)}
\end{gathered}
$$

The flux is $\Phi=4 \pi \lambda / e$.

Again types 2 and 3 give one parameter families, and type 4 a two parameter family. There are clear relations between the types of solution here and the corresponding types above. (Furthermore the similarity extends to solutions with singularities of the two equations, that are not of physical interest.) We note that requiring singlevaluedness of $A_{i}$ at the origin in the case we have just discussed would produce a requirement that $p$ should be an integer; this is not reasonable from a physical standpoint, however, since for our second choice of $b$ the origin is a singular point, and therefore there is no justification for imposing single-valuedness there. $p$ would similarly be quantized if we insisited that the Euler character associated with our metric should be an integer, but there is no reason to do this either (the metric we have is flat everywhere but at the origin, and in fact gives space the topology of a cylinder). It would be very interesting to elucidate further the relation of the two sets of solutions above, and understand how to 'perturb' any ODE with the Painlevé property in such a way as to keep the qualitative nature of the solutions. It seems that all we lose going from the integrable to non-integrable cases is the ability to explicitly write down solutions.

To return to the PDE $(\mathrm{J})$, or equivalently the system $(\mathrm{F})$, once we have shown the existence of one solution, it is possible to use index theory methods to determine how we can vary that solution to obtain other solutions of the system. For the flat space case one can show that there are $2 n$ deformations of the n-vortex solution. On the 
other hand it is possible to show that equation $(\mathrm{J})$ does not have the Weiss-TaborCarnevale Painlevé property for any choice of $b$. It would seem that equation $(J)$ furnishes an example of a non-integrable system with soliton-type solutions (which can not be explicitly written down).

I wish to devote the rest of this presentation to dealing with some interesting, if not very novel, aspects of Painleve analysis that arose in the analysis of equation (L) for arbitrary $b$. In fact let us do the Painlevé analysis (in the style of $[\mathrm{F}]$ ) for the ODE

$$
\frac{1}{r}\left(\frac{r \chi^{\prime}}{\chi}\right)^{\prime}=f \chi^{2}+g \chi
$$

where $f, g$ are arbitrary functions of $r$. We write this in the form

$$
\chi \chi^{\prime \prime}-\chi^{\prime 2}+\frac{\chi \chi^{\prime}}{r}=f \chi^{4}+g \chi^{3}
$$

and look for a solution in the form

$$
\chi=\sum_{n=0}^{\infty} \chi_{n}\left(r-r_{0}\right)^{n-1}
$$

Remembering to expand $f, g, 1 / r$ in Taylor series around $r_{0}$,

$$
f(r)=\sum_{n=0}^{\infty} f_{n}\left(r-r_{0}\right)^{n} \quad f_{n}=\left.\frac{1}{n !} \frac{d^{n} f}{d r^{n}}\right|_{r=r_{0}}
$$

etc., we obtain at leading order

$$
\chi_{0}^{2}=\frac{1}{f_{0}}
$$

We find that resonances occur at $n=-1,2$ and the consistency condition for the $n=2$ resonance is

$$
\left( \pm \frac{2 g r}{\sqrt{f}}+\frac{f^{\prime} r}{f}\right)^{\prime}=0
$$

The \pm in $(\mathrm{U})$ originates from the fact that $(\mathrm{T})$ allows two choices for $\chi_{0}$; Painlevé property only holds if (U) is satisfied for both sign choices, implying we need

$$
\begin{aligned}
& f=A r^{B} \\
& g=C r^{\frac{1}{2} B-1}
\end{aligned}
$$

where $A, B, C$ are arbitrary constants. (for $f=-g=\gamma^{2} b$ we need $B=-2, A=-C$ giving $b$ as taken above). If we insist on consistency for just one choice of sign we need

$$
\begin{aligned}
& f=h^{2} r^{-2 D} \\
& g=h^{\prime} r^{-D}
\end{aligned}
$$

where $h$ is an arbitrary function and $D$ is an arbitrary constant. I wish to show that for this more general choice of $f, g$ it is possible to find a one parameter family of solutions (unfortunately they do not have the boundary conditions required for our physical application); this emerges in the course of rewriting equation ( $\mathrm{R}$ ) (for the choice (W)) in a form where instead of having two different possible residues (the two possible values of $\chi_{0}$ ) there are two different leading orders in the Painlevé analysis (this will become apparent). 
We proceed as follows. In equation $(\mathrm{R})$, with the choice $(\mathrm{W})$, we make successive changes in the independent variable, dependent variable and arbitrary function as follows:

$$
\begin{aligned}
t & =\ln r \\
Y & =\chi h^{-1} e^{(D-1) t} \\
v & =\dot{h} / h
\end{aligned}
$$

(dots denore differentiation with respect to $t$ ). Our equation becomes

$$
\frac{d^{2}}{d t^{2}}(\ln Y)=Y^{2}+v Y+\dot{v}
$$

We now make the crucial substitution $Y=\dot{q} / q$; where $Y$ has a pole with a positive (negative) residue, $q$ will have a zero (pole). We obtain a third order equation, which can however be integrated once to yield

$$
\ddot{q}=\frac{2 \dot{q}^{2}}{q}+v \dot{q}+\frac{\epsilon \dot{q}}{q}
$$

where $\epsilon$ is an arbitrary constant. Setting $r=1 / q$ this becomes

$$
\ddot{r}=v \dot{r}+\epsilon r \dot{r}
$$

For $\epsilon=0$ this can be solved to give

$$
r=F+G \int d t \exp \left(\int d t v\right)
$$

where $F, G$ are constants of integration. This yields a one parameter family of solutions $\chi$ to the original equation. For $\epsilon \neq 0$, Painlevé analysis of equation (AA) requires looking at two expansions

$$
\begin{aligned}
& r=\sum_{n=0}^{\infty} r_{n}\left(t-t_{0}\right)^{n-1} \\
& r=\sum_{n=0}^{\infty} r_{n}\left(t-t_{0}\right)^{n+1}
\end{aligned}
$$

so we might describe the procedure we have followed as 'resolving two residues into two different leading orders'. An expansion of form (DD) is consistent for any $v$ in (AA), but an expansion of form (CC) is only consistent if $v$ is a constant.

The reason I have detailed this at such length is that it seems that it may well be useful in other circumstances; whenever an equation has two possible leading coefficients in its Painlevé expansion (for a particular leading order), it can, by a suitable substution, increasing the order of the ODE by one, be brought to a form where the two possible leading coefficients have been 'resolved' into two possible leading orders. Integrating this higher-order equation once, if possible, will introduce an arbitrary constant, and for a special value of this constant we may be able to extract special solutions. For example, for the second Painlevé equation

$$
w^{\prime \prime}=2 w^{3}+w r+\alpha
$$

the Painlevé expansions starts 


$$
w=\frac{ \pm 1}{r-r_{0}}+\ldots
$$

To 'resolve' the \pm we set $w=u^{\prime} / u$ to obtain

$$
\left(\frac{u^{\prime \prime}}{u^{3}}+\frac{r}{2 u^{2}}\right)^{\prime}=\frac{\left(\alpha+\frac{1}{2}\right)}{u^{2}}
$$

For $\alpha=-\frac{1}{2}$ this can be integrated to

$$
u^{\prime \prime}+\frac{1}{2} r u=C u^{3}
$$

where $C$ is an arbitrary constant; for $C=0$ we obtain at once the special solutions of the $\alpha=-\frac{1}{2}$ second Painlevé equation, in terms of solutions of Airy's equation.

To return to our main subject, and conclude, we have seen that the ChernSimons-Higgs vortex equations provide a system that, while not integrable, seems, in some non-formal sense, to be very close to an integrable system. For the cylindrically symmetric case we can see explicitly how solutions for the flat space case mimic solutions for the integrable case. Many similar results to the ones we have presented here can be presented for the more standard Abelian Higgs vortex equations (see the references in $[C]$ and $[D])$ : for flat space the relevant PDE fails the Painlevé test, despite the existence of $n$-vortex solutions. It is to be hoped that the relationship of the vortex systems to usual integrable systems might be understood better.

\section{A REDUCTION OF THE SELF-DUAL YANG-MILLS EQUATIONS TO THREE DIMENSIONS}

\subsection{INTRODUCTION}

As we have already heard in this workshop, in the talk of Ablowitz [1], there has been some recent interest in the self-dual Yang-Mills (SDYM) equations, as it seems that a large number of known integrable systems (in $0+1$ and $1+1$ dimensions) can be obtained by reduction of the SDYM equations, with a suitable choice of the gauge group. That reductions of the SDYM equations are integrable is no surprise, as the SDYM equations themselves can be completely solved [2]. Furthermore the SDYM equations possess a host of the 'standard properties' of integrable systems: they have Painlevé property (shown in the sense of Weiss, Tabor and Carnevale (WTC) for gauge group $S U(2)$ in [3], and in a possibly stronger sense for arbitrary gauge group in [4]), they have an infinite number of conservation laws associated with them [5], they have Bäcklund transformations [6], and they can be written as consistency conditions for a system of linear equations [7]. Any reduction of the SDYM equations will inherit all these properties. It is of interest to ask what integrable systems can arise by reduction from the SDYM equations. Any system in 1+1 dimensions that can written as a single zero curvature condition, can be obtained from SDYM, as we shall see shortly, and this includes many systems, such as the KdV, mKdV, NLS, Sine-Gordon, Boussinesq [8], Super-KdV [9] and Toda chain [10] equations. By further reduction of these systems it follows that one can obtain a number of the Painlevé equations in $0+1$ dimensions, and Ablowitz et. al. [1] have shown how to obtain other interesting $0+1$ dimensional systems. Here we shall investigate briefly a $2+1$ dimensional reduction of SDYM. One cannot expect to obtain the KP or Davey-Stewartson equations from SDYM (at least with a finite dimensional gauge group), because these equations would thereby inherit a Lax pair formalism of a type they are believed not to have. But it is nevertheless of interest to see what one does obtain. We will follow a simple reduction technique, which is a trivial generalization of a method recently used by Mason and Sparling [11] to obtain KdV and NLS from SDYM. We obtain equations which superficially are $2+1$ dimensional, but actually have the structure of $1+1$ dimensional systems, as we 
will explain. Despite this, it is still of interest to see the equations emerge explicitly from SDYM. We also demonstrate some interesting properties for the simplest case (KdV-type) of our reduction scheme.

\subsection{REDUCTION METHOD}

The SDYM equations can be presented as follows [12]: let $A_{z}, A_{\bar{z}}, A_{w}, A_{\bar{w}}$ be functions of the variables $z, \bar{z}, w, \bar{w}$ taking values in a Lie algebra $\mathcal{G}$. For $i, j \in\{z, \bar{z}, w, \bar{w}\}$, $i \neq j$ define

$$
F_{i j}=\partial_{i} A_{j}-\partial_{j} A_{i}+\left[A_{i}, A_{j}\right]
$$

The SDYM equations are

$$
\begin{aligned}
F_{z w} & =0 \\
F_{w \bar{w}}+F_{z \bar{z}} & =0 \\
F_{\bar{z} \bar{w}} & =0
\end{aligned}
$$

Choosing the coordinates $w, \bar{w}, z, \bar{z}$ to be complex conjugate pairs in the obvious manner, is appropriate for the SDYM equations on flat Euclidean $\mathbf{R}^{4}$; for this talk however we shall take all the coordinates to be real, which is appropriate for the SDYM equations on flat $\mathbf{R}^{4}$ with a metric of signature $(2,2)$. The SDYM equations are invariant under gauge transformations $A_{i} \rightarrow g A_{i} g^{-1}+g \partial_{i} g^{-1}$, where $g$ is a function with values in the Lie group associated with $\mathcal{G}$. We use this freedom to set $A_{\bar{z}}$ to zero; we still have the residual freedom of gauge transformations with $g$ independent of $\bar{z}$. We now perform a reduction to three dimensions by taking all the remaining functions independent of $\bar{w}$. The $F_{\bar{z} \bar{w}}=0$ equation becomes, as a result of our gauge choice,

$$
\partial_{\bar{z}} A_{\bar{w}}=0
$$

Since $A_{\bar{w}}$ is independent of $\bar{z}$, it follows that we can apply the residual gauge transformations to it to bring it to a canonical form of some sort. Indeed, if we had not performed the reduction, then $A_{\bar{w}}$ would transform by $A_{\bar{w}} \rightarrow g A_{\bar{w}} g^{-1}+g \partial_{\bar{w}} g^{-1}$ and we would be able to gauge transform $A_{\bar{w}}$ to zero. But because of the reduction, $A_{\bar{w}}$ transforms homogeneously, $A_{\bar{w}} \rightarrow g A_{\bar{w}} g^{-1}$, and therefore it cannot be taken to be zero, but can be taken to be one of several canonical forms (all independent of $\bar{z}, \bar{w}$, of course). We shall not here attempt to classify these forms, but shall focus on the simplest examples. The remaining equations we have to solve are

$$
\begin{aligned}
\partial_{z} A_{w}-\partial_{w} A_{z}+\left[A_{z}, A_{w}\right] & =0 \\
\partial_{w} A_{\bar{w}}+\left[A_{w}, A_{\bar{w}}\right]-\partial_{\bar{z}} A_{z} & =0
\end{aligned}
$$

The first of these is a zero curvature condition for $A_{z}, A_{w}$; the second is a simple linear relation between $A_{z}$ and $A_{w}$ for a given canonical choice of $A_{\bar{w}}$. Note that if we choose $A_{\bar{w}}=0$ and further reduce by taking $A_{z}, A_{w}$ independent of $\bar{z}$, then we obtain just a single zero curvature condition for $A_{z}(z, w), A_{w}(z, w)$, and, as mentioned in the introduction, many integrable systems arise this way: for example, for KdV one chooses (with $\mathcal{G}=\operatorname{sl}(2, \mathbf{C})$ )

$$
A_{z}=\left(\begin{array}{cc}
0 & 1 \\
-u & 0
\end{array}\right) \quad A_{w}=\frac{1}{4}\left(\begin{array}{cc}
-u_{z} & 2 u \\
-\left(2 u^{2}+u_{z z}\right) & u_{z}
\end{array}\right)
$$

(where $u$ is a function of $z$ and $w$ ). Note further that in equations (4) the coordinate $\bar{z}$ appears on a different footing from $z, w$. The same observation can be made on two other results: first, if we apply our reduction to the standard Lax pair formulation of SDYM, we see our equations can be written

$$
\left[\partial_{z}+A_{z}+\lambda A_{\bar{w}}, \partial_{w}+\lambda \partial_{\bar{z}}+A_{w}\right]=0
$$


(Here $\lambda$ is a parameter, and our equations emerge by requiring the above to be true order-by-order in $\lambda$ ). Note in this that if we define $\bar{Z}=\bar{z}-\lambda w, W=w$, then the derivative with respect to $\bar{Z}$ does not appear in the Lax pair. Second, it is straightforward to show how to reduce the standard non-local conservation laws for SDYM [5], to obtain the existence of quantities $V^{(n)}(z, \bar{z}, w), n=0,1,2, \ldots$ such that

$$
\partial_{w}\left(\int d z V^{(n)}\right)=0
$$

(Here the quantities $V^{(n)}$ are non-local in the $A$ 's). In these conservation laws, $\bar{z}$ has a parametric role. We now proceed to give explicitly the simplest examples of the reduction, and to explain the observation that one coordinate seems to be redundant.

\subsection{EXAMPLE 1}

We choose $\mathcal{G}=\operatorname{sl}(2, \mathbf{C})$, and

$$
A_{\bar{w}}=\left(\begin{array}{ll}
0 & 0 \\
1 & 0
\end{array}\right)
$$

We parametrize $A_{z}, A_{w}$ by

$$
A_{w}=\left(\begin{array}{cc}
\alpha & \beta \\
\tilde{\beta} & -\alpha
\end{array}\right) \quad A_{z}=\left(\begin{array}{cc}
\gamma & \delta \\
\tilde{\delta} & -\gamma
\end{array}\right)
$$

and insert into equations (4). We meet the equations

$$
\begin{gathered}
\partial_{\bar{z}} \delta=0 \\
\partial_{\bar{z}}\left(\tilde{\delta}+\gamma_{z}+\gamma^{2}\right)=0
\end{gathered}
$$

which we solve (for simplicity) by taking $\delta=1$ and $\tilde{\delta}=-\left(\gamma_{z}+\gamma^{2}\right)$. We obtain

$$
A_{w}=\left(\begin{array}{cc}
\frac{1}{2} \gamma_{z \bar{z}}+\gamma \gamma_{\bar{z}} & \gamma_{\bar{z}} \\
\tilde{\beta} & -\left(\frac{1}{2} \gamma_{z \bar{z}}+\gamma \gamma_{\bar{z}}\right)
\end{array}\right) \quad A_{z}=\left(\begin{array}{cc}
\gamma & 1 \\
-\left(\gamma_{z}+\gamma^{2}\right) & -\gamma
\end{array}\right)
$$

where

$$
\tilde{\beta}=\gamma_{w}-\frac{1}{2} \gamma_{z z \bar{z}}-\gamma \gamma_{z \bar{z}}-2 \gamma_{z} \gamma_{\bar{z}}-\gamma^{2} \gamma_{\bar{z}}
$$

and $\gamma$ satisfies the equation

$$
\gamma_{w z}=\frac{1}{4} \gamma_{z z z \bar{z}}+2 \gamma_{z} \gamma_{z \bar{z}}+\gamma_{z z} \gamma_{\bar{z}}
$$

This equation is our result. Setting $u=\gamma_{z}$ we can write this

$$
\begin{aligned}
u_{w} & =\frac{1}{4} u_{z z \bar{z}}+2 u u_{\bar{z}}+u_{z}\left(\int d z u_{\bar{z}}\right) \\
& =\left(\frac{1}{4} \partial_{z}^{2}+2 u+\left(\partial_{z} u\right) \partial_{z}^{-1}\right) u_{\bar{z}}
\end{aligned}
$$

We see that our equation is simply $u_{w}=\Phi(u) u_{\bar{z}}$ where $\Phi(u)$ is the recursion operator of the KdV equation.* Our equation, while being an integrable differential equation in three dimensions, has essentially the same structure as the $\operatorname{KdV}$ equation $\left(u_{w}=\right.$ $\left.\Phi(u) u_{z}\right)$, in the same way that all the higher $\mathrm{KdV}$ equations $\left(u_{w}=\Phi(u)^{n} u_{z}, n=\right.$

\footnotetext{
* I thank A.S.Fokas for this critical observation.
} 
$2,3, \ldots)$ have the same structure as the KdV equation. For all these equations, the integrability arises as a consequence of the existence of the 'strong symmetry' $\Phi$ (see [13] for more details). It seems likely that all the integrable equations that arise from our reduction will be three dimensional analogues of two dimensional integrable systems in this way, thereby explaining the observations at the end of section 2 . We note that if we set $z=\bar{z}$ in (15) we obtain the $\mathrm{KdV}$ equation; this is the reduction of Mason and Sparling [11].

Despite the fact that the equation (14) we have obtained is not a new type of integrable equation, we establish some of its properties here.

1. Equation (14) has the Painlevé property in the sense of WTC. This is straightforward to check, using a reduced ansatz

$$
\begin{gathered}
\gamma=\sum_{n=0}^{\infty} \gamma_{n}(\bar{z}, w) \phi^{n-1} \\
\phi=z+\alpha(\bar{z}, w)
\end{gathered}
$$

The resonances are at $n=-1,1,4,6$ (i.e. $\alpha, \gamma_{1}, \gamma_{4}, \gamma_{6}$ are arbitrary functions); these are the same as for the KP equation in the form

$$
v_{x t}+v_{x x x x}+v_{x} v_{x x}+v_{y y}=0
$$

(obtained from the usual form of the KP equation by setting $u=v_{x}$ ).

2. For the KdV equation, truncating the Painlevé expansion to contain just singular terms produces the substitution necessary to pass to Hirota bilinear form [14]. For equation (14) Painlevé analysis suggests the substitution

$$
\gamma=\frac{\phi_{z}}{\phi}
$$

This yields

$$
\begin{aligned}
0= & 4\left(\phi^{2} \phi_{z z w}-\phi \phi_{z z} \phi_{w}-2 \phi \phi_{z} \phi_{w z}+2 \phi_{z}^{2} \phi_{w}\right) \\
& +\phi^{2} \phi_{z z z z \bar{z}}-\phi \phi_{\bar{z}} \phi_{z z z z}-4 \phi \phi_{z} \phi_{z z z \bar{z}}+2 \phi \phi_{z z} \phi_{z z \bar{z}} \\
& +4 \phi_{z} \phi_{\bar{z}} \phi_{z z z}-2 \phi_{\bar{z}} \phi_{z z}^{2}-4 \phi_{z} \phi_{z z} \phi_{z \bar{z}}+4 \phi_{z}^{2} \phi_{z z \bar{z}}
\end{aligned}
$$

This appears to be in some sort of trilinear form. It seems reasonable to attempt to find some suitable extension of Hirota's bilinear operators to cast the above expression into a simpler form. ${ }^{*}$ To this end, define the action of the trilinear operator $\mathcal{D}_{z}^{m} \mathcal{D}_{\bar{z}}^{n} \mathcal{D}_{w}^{p}$ on a triplet of functions $(a, b, c)$ (all functions of $z, \bar{z}, w)$ by

$$
\begin{aligned}
\mathcal{D}_{z}^{m} \mathcal{D}_{\bar{z}}^{n} \mathcal{D}_{w}^{p}(a, b, c)= & \left(\frac{\partial}{\partial z}+\omega \frac{\partial}{\partial z^{\prime}}+\omega^{2} \frac{\partial}{\partial z^{\prime \prime}}\right)^{m}\left(\frac{\partial}{\partial \bar{z}}+\omega^{2} \frac{\partial}{\partial \bar{z}^{\prime}}+\omega \frac{\partial}{\partial \bar{z}^{\prime \prime}}\right)^{n} \\
& \left(\frac{\partial}{\partial w}+\omega \frac{\partial}{\partial w^{\prime}}+\omega^{2} \frac{\partial}{\partial w^{\prime \prime}}\right)^{p} \\
& \left.a(z, \bar{z}, w) b\left(z^{\prime}, \bar{z}^{\prime}, w^{\prime}\right) c\left(z^{\prime \prime}, \bar{z}^{\prime \prime}, w^{\prime \prime}\right)\right|_{(w, z, \bar{z})=\left(w^{\prime}, z^{\prime}, \bar{z}^{\prime}\right)=\left(w^{\prime \prime}, z^{\prime \prime}, \bar{z}^{\prime \prime}\right)}
\end{aligned}
$$

* I thank J. Hietarinta for pointing out to me that equation (14) can actually be written using Hirota's bilinear operators, by the introduction of an extra independent variable, as explained in [15]; this method is also used for bilinearisation of higher order KdV equations [16], and hence is certainly appropriate here. I also thank J. Hietarinta for pointing out to me reference [17], where another type of trilinear form is introduced. 
Here $\omega$ is a cube root of unity obeying $\omega+\omega^{2}=-1$; note the asymmetry between $z$ and $\bar{z}$ in this formula. With this definition our equation can be written in the elegant form

$$
\left(4 \mathcal{D}_{z}^{2} \mathcal{D}_{w}-\mathcal{D}_{z}^{4} \mathcal{D}_{\bar{z}}\right)(\phi, \phi, \phi)=24\left|\begin{array}{ccc}
\phi & \phi_{z} & \phi_{\bar{z}} \\
\phi_{z} & \phi_{z z} & \phi_{z \bar{z}} \\
\phi_{z z} & \phi_{z z z} & \phi_{z z \bar{z}}
\end{array}\right|
$$

3. The final form of the equation given in the last paragraph turns out to be convenient for finding 1- and 2-soliton solutions. Defining $\lambda_{i}=\alpha_{i} z+\beta_{i} \bar{z}+\gamma_{i} w+\delta_{i}, i=1,2$, with $\gamma_{i}=\alpha_{i}^{2} \beta_{i} / 4$, the general 2-soliton can be written

$$
\phi=1+e^{\lambda_{1}}+e^{\lambda_{2}}+\eta e^{\lambda_{1}+\lambda_{2}}
$$

where

$$
\eta=\left(\frac{\alpha_{1}-\alpha_{2}}{\alpha_{1}+\alpha_{2}}\right)^{2}
$$

The fact that the phase shift can be written purely in terms of $\alpha_{1}, \alpha_{2}$ is presumably the expression of the underlying two-dimensional structure of our equation.

4. Just as the AKNS formalism for the KdV equation can be reduced to a scalar scattering problem, for this particular reduction of SDYM, the SDYM Lax pair can be reduced to solving the scalar equations:

$$
\begin{gathered}
\left(\partial_{z}^{2}+2 \gamma_{z}\right) \psi=\lambda \psi \\
\left(\partial_{w}-\gamma_{\bar{z}} \partial_{z}+\frac{1}{2} \gamma_{z \bar{z}}\right) \psi=-\lambda \partial_{\bar{z}} \psi
\end{gathered}
$$

Once again we see we have a KdV theory, as the scattering problem that has to be solved is just that of the Schrödinger equation. We note that if we define

$$
\begin{aligned}
L & =\partial_{z}^{2}+2 \gamma_{z} \\
M & =-\gamma_{\bar{z}} \partial_{z}+\frac{1}{2} \gamma_{z \bar{z}}
\end{aligned}
$$

then our equation can be written

$$
L_{w}=[L, M]+2 \gamma_{z \bar{z}} L
$$

\subsection{EXAMPLE 2}

For our second example we again take $\mathcal{G}=\operatorname{sl}(2, \mathbf{C})$, and now

$$
A_{\bar{w}}=\kappa\left(\begin{array}{cc}
1 & 0 \\
0 & -1
\end{array}\right)
$$

After one integration one obtains the system of equations

$$
\begin{aligned}
\chi_{z} & =(\delta \tilde{\delta})_{\bar{z}} \\
2 \kappa \delta_{w} & =-\delta_{z \bar{z}}+2 \delta \chi \\
-2 \kappa \tilde{\delta}_{w} & =-\tilde{\delta}_{z \bar{z}}+2 \tilde{\delta} \chi
\end{aligned}
$$

A priori the functions $\chi, \delta, \tilde{\delta}$ are all complex, but we can consistently take $\tilde{\delta}$ to be plus or minus the complex conjugate of $\delta$ if $\kappa$ is pure imaginary. Then, setting $\kappa=i k$ we have

$$
-2 k \delta_{w}=\left(-i \partial_{z} \pm 4 i \delta \partial_{z}^{-1} \operatorname{Re}(\bar{\delta} \cdot)\right) \delta_{\bar{z}}
$$


i.e. $-2 k \delta_{w}=\Psi(\delta) \delta_{\bar{z}}$, where $\Psi(\delta)$ is the recursion operator for the NLS equation [13] (NLS is obtained from our system by setting $z=\bar{z}[11]$ ). Thus our system here is related to NLS in exactly the same way that the system obtained in section 3 is related to KdV. We note that the fifth order system obtained here has WTC Painlevé property, with resonances $n=-1,0,2,3,4$.

\subsection{CONCLUSION}

The essential result we have presented here is that a simple generalization of Mason and Sparling's [11] reduction of SDYM to two dimensional integrable systems yields three dimensional integrable systems with essentially the same structure as the two dimensional integrable systems; in effect, removing the 'timelike symmetry' (i.e. dependence on $z, \bar{z}$ only through $z+\bar{z}$ ) imposed by Mason and Sparling has no consequence. We note that Fokas and Santini [18] have recently shown that the recursion operators of $\mathrm{KdV}$, of KP, of SDYM and of Chiral Fields are all in fact concrete realizations of the same abstract recursion operator. It is clearly of interest to clarify the structure of SDYM further and to see how it 'contains' and 'unifies' many two dimensional integrable systems, and their extensions.

\section{ACKNOWLEDGEMENTS}

I thank V.P.Nair and M.Tabor for discussions and encouragement through this work. For my second presentation, I am much indebted to A.S.Fokas for clarifying the nature of the three dimensional systems I obtained, and I also thank M.J.Ablowitz for some comments. I thank V.P.Nair for a critical reading of the manuscript, and A.S.Fokas and M.Tabor for a critical reading of the second part of the manuscript. This work was supported in part by the U.S.Department of Energy.

\section{REFERENCES}

[A] R.Jackiw and S.Templeton, Phys.Rev.D 23 (1981) 2291; J.Schonfeld, Nucl.Phys. B185 (1981) 157; S.Deser, R.Jackiw and S.Templeton, Phys.Rev.Lett. 48 (1982) 975, Ann.Phys. 140 (1982) 372, (E) 185 (1988) 406.

[B] S.Deser and Z.Yang, Mod.Phys.Lett.A 4 (1989) 2123.

[C] J.Hong, Y.Kim and P.Y.Pac, Phys.Rev.Lett. 64 (1990) 2230; R.Jackiw and E.J.Weinberg, Phys.Rev.Lett. 64 (1990) 2234; R.Jackiw, K.Lee and E.J.Weinberg, "Self-Dual Chern-Simons Solitons", Phys.Rev.D., to appear.

[D] J.Schiff, "Integrability of Chern-Simons-Higgs and Abelian Higgs Vortex Equations in a Background Metric", J.Math.Phys., to appear.

[E] H.Airault, Stud.Appl.Math 61 (1979) 31.

[F] M.J.Ablowitz, A.Ramani and H.Segur J.Math.Phys. 21 (1980) 715.

[1] See the talk of M.J.Ablowitz at this workshop, and S.Chakravarty, M.J. Ablowitz and P.A.Clarkson, Phys.Rev.Lett. 65 (1990) 1085.

[2] M.F.Atiyah, N.J.Hitchin, V.G.Drinfeld and Yu.I.Manin, Phys.Lett. 65A (1978) 185.

[3] M.Jimbo, M.D.Kruskal and T.Miwa, Phys.Lett. 92A (1982) 59; R.S.Ward Nonlinearity 1 (1988) 671.

[4] R.S.Ward, Phys.Lett. 102A (1984) 279.

[5] See, for example, L.Dolan, Phys.Rep. 109 (1984) 1; L.L.Chau in Integrable Systems, M.L.Ge and X.C.Song (eds.), World Scientific, (1990).

[6] E.Corrigan, D.B.Fairlie, R.G.Yates and P.Goddard, Phys.Lett. 72B (1978) 354, Comm.Math.Phys. 58 (1978) 223.

[7] A.A.Belavin and V.E.Zakharov, Phys.Lett. 73B (1978) 53.

[8] I.M.Krichever and S.P.Novikov, Physica 3D (1981) 267.

[9] A.Das and S.Roy, University of Rochester preprint, UR-1137. 
[10] A.N.Leznov and M.V.Saveliev, Lett.Math.Phys. 3 (1979) 489.

[11] L.J.Mason and G.A.J.Sparling, Phys.Lett.A 137 (1989) 29.

[12] C.N.Yang, Phys.Rev.Lett. 38 (1977) 1377.

[13] B.Fuchssteiner and A.S.Fokas, Physica 4D (1981) 47.

[14] J.D.Gibbon, P.Radmore, M.Tabor, D.Wood, Stud. in Appl.Math 72 (1985) 39.

[15] J.Hietarinta, in Partially Integrable Evolution Equations in Physics, R.Conte and N.Boccara (eds.), Kluwer Academic Publishers, the Netherlands (1990).

[16] P.G.Drazin and R.S.Johnson, Solitons: An Introduction, Cambridge University Press (1989).

[17] J.Matsukidaira,J.Satsuma and W.Strampp, Phys.Lett.A 147 (1990) 467.

[18] A.S.Fokas and P.M.Santini, in Solitons in Physics, Mathematics and Nonlinear Optics, P.J.Olver and D.H.Sattinger (eds.), Springer Verlag (1990). 